MS \#AJG-06-2061-R2

\title{
Association of Autoimmune Pancreatitis with Cytotoxic T-lymphocyte Antigen 4 Gene Polymorphisms in Japanese Patients
}

Short title: CTLA4 Polymorphisms in Japanese AIP

\begin{abstract}
Takeji Umemura, M.D., Ph.D., ${ }^{1}$ Masao Ota, Ph.D., ${ }^{2}$ Hideaki Hamano, M.D., Ph.D., ${ }^{1}$ Yoshihiko Katsuyama, Ph.D., ${ }^{3}$ Takashi Muraki, M.D., Ph.D., ${ }^{1}$ Norikazu Arakura, M.D., Ph.D., ${ }^{1}$ Shigeyuki Kawa, M.D., Ph.D., ${ }^{4}$ and Kendo Kiyosawa, M.D., Ph.D. ${ }^{1}$
\end{abstract}

Departments of ${ }^{1}$ Internal Medicine, Division of Gastroenterology and Hepatology, ${ }^{2}$ Department of Legal Medicine, ${ }^{3}$ Department of Pharmacy, Shinshu University School of Medicine, Matsumoto, Japan, ${ }^{4}$ Center for Health, Safety and Environmental Management, Shinshu University, Matsumoto, Japan

Key Words: CTLA4, autoimmune pancreatitis, HLA, disease susceptibility.

Corresponding author: Takeji Umemura, MD, PhD, Department of Internal Medicine, Shinshu University School of Medicine, 3-1-1 Asahi, Matsumoto 390-8621, Japan.

E-mail: t-ume@hsp.md.shinshu-u.ac.jp; Telephone: +81-263-37-2634;

Fax: $+81-263-32-9412$.

Word count abstract: 250 and text: 2834

CTLA4 Polymorphisms in Japanese AIP 


\begin{abstract}
Objectives: Autoimmune pancreatitis (AIP) is characterized by high serum IgG4 concentrations, lymphoplasmacytic inflammation, and a favorable response to corticosteroid treatment. Although the HLA DRB $1^{*} 0405-\mathrm{DQB} 1 * 0401$ haplotype and Fc receptor-like 3 polymorphisms have been associated with AIP, the role of other genetic factors is largely unknown. As cytotoxic T lymphocyte antigen 4 (CTLA4) polymorphisms have recently been linked with several autoimmune diseases, we sought to determine if CTLA4 polymorphisms and serum SCTLA4 levels were associated with AIP as well.

Methods: Five CTLA4 polymorphisms, located at $-1722,-658$, and -318 in the promoter, +49 in exon 1 , and +6230 in the $3^{\prime}$ untranslated region, were genotyped in 59 patients with AIP and 102 healthy subjects. Serum sCTLA4 levels were also determined in cohorts of 52 patients and 32 controls. Results: Compared with healthy subjects, we found a significant increase in the $+6230 \mathrm{G} / \mathrm{G}$ genotype $(64 \%$ vs. $42 \%$, odds ratio [OR], $2.48 ; P=$ 0.011 ) in AIP patients. Haplotype 2 , which had the $+6230 \mathrm{~A}$, was associated with AIP resistance (OR, 0.49; $P=0.011)$. The $+49 \mathrm{~A} / \mathrm{A}$ and $+6230 \mathrm{~A} / \mathrm{A}$ genotypes were associated with an enhanced risk of relapse (OR, 5.45; $P=0.038$ and OR, 12.66; $P=0.022$ ). Additionally, median serum SCTLA4 levels were significantly higher in patients with AIP $(8.9 \mathrm{ng} / \mathrm{mL})$ compared with healthy subjects $(2.9 \mathrm{ng} / \mathrm{mL} ; P<0.001)$. The $+6230 \mathrm{G} / \mathrm{A}$ polymorphism did not influence sCTLA4 levels in AIP patients. Conclusions: Our findings suggest that AIP is associated with a genetic polymorphism in CTLA4 and is positively correlated with serum sCTLA4 levels.
\end{abstract}


STUDY HIGHLIGHTS

1) What Is Current Knowledge

- The HLA DRB1 ${ }^{*} 0405-\mathrm{DQB} 1{ }^{*} 0401$ haplotype and Fc receptor-like gene 3 polymorphisms are associated with autoimmune pancreatitis (AIP) in the Japanese population.

2) What Is New Here

- The cytotoxic T lymphocyte antigen $4(C T L A 4)+6230 \mathrm{G} / \mathrm{G}$ genotype is significantly associated with AIP.

- The +49A/A and +6230A/A genotypes are associated with an enhanced risk of relapse.

- $\quad$ sCTLA4 levels are significantly higher in patients with AIP compared with healthy subjects. 


\section{INTRODUCTION}

Autoimmune pancreatitis (AIP) is a unique form of chronic pancreatitis characterized by male preponderance, irregular narrowing of the main pancreatic duct, swelling of the pancreatic parenchyma, histological evidence of lymphoplasmacytic inflammation, and a favorable response to corticosteroid treatment (1-6). This disease is also characterized by systemic complications involving various extra-pancreatic lesions of similar lymphoplasmacytic inflammation, and has thus been recognized as a systemic inflammatory condition (7-12). In this regard, considerable attention has been drawn to the clinical importance of this disease, and the pathogenesis and role of genetic factors have been extensively explored. We and others have previously reported that IgG4 concentrations are significantly and specifically higher in patients with AIP, suggesting that IgG4 plays a major role in pathogenesis $(13,14)$. The HLA DRB1*0405-DQB1*0401 haplotype has been identified as the main determinant of AIP susceptibility in the Japanese population (15), along with Fc receptor-like gene 3 (FCRL3) polymorphisms, which lie outside the major histocompatibility complex region (16). However, because none of the genetic markers identified so far are sufficient to fully explain disease etiology, additional genes with influences on immunologic tolerance are likely to be present.

One of the characteristic pathological features in AIP is lymphoplasmacytic infiltration, which includes CD4 or CD8 T lymphocytes and IgG4-bearing plasma cells in the pancreatic parenchyma and other involved organs $(7,8,12,17)$. Thus, factors regulating $T$ cell function may also influence the development of AIP. The cytotoxic T lymphocyte antigen 4 (CTLA4; CD152) gene is an inhibitory receptor expressed on the cell surface of activated memory $\mathrm{T}$ cells and on $\mathrm{CD} 4^{+} \mathrm{CD} 25^{+}$regulatory $\mathrm{T}$ cells, and acts largely as a negative regulator of $T$ cell responses (18). CTLA4 seems to modulate positive $T$ cell costimulatory signals by competing with the CD28 molecule for engagement with the B7 molecules CD80 and CD86 that are found on antigen presenting cells. CTLA4-deficient mice develop lymphoproliferative disorders with multiorgan lymphocytic infiltration (19). Specifically, CTLA4 +49A/G single nucleotide polymorphisms (SNPs) have been associated with susceptibility to autoimmune diseases, such as type 1 diabetes, autoimmune thyroid disease, autoimmune hepatitis, and primary biliary cirrhosis (18).

Recent studies have revealed an alternative splice form of CTLA4, secreted by resting T cells, can suppress $T$ cell activation. This soluble isoform of CTLA4 (sCTLA4) is present in human serum, and is elevated in patients with autoimmune diseases, such as autoimmune thyroid disease (20), systemic lupus erythematosus (21), and myasthenia gravis (22). Furthermore, the $+6230 \mathrm{G} / \mathrm{A}$ SNP in the 3' untranslated region of CTLA4 was found to be associated with Graves' disease, type 1 diabetes (23), and clearance of hepatitis B virus (24). However, this gene has not been previously examined with respect to AIP.

Therefore, in the present study, we investigated the association of AIP disease susceptibility and four haplotype-tagging SNPs and the SNP at +6230 of CTLA4 in the Japanese population. Furthermore, we sought to determine whether or not serum sCTLA4 levels were varied with the genotypes of CTLA4 in the AIP patients.

\section{PATIENTS AND METHODS}

\section{Subjects}


Between September 1994 and October 2006, serum samples were obtained from 59 patients with AIP (49 men and 10 women), aged 38-76 years (median 63 years), and 102 healthy control subjects. All control subjects had indicated the absence of major illness on a standard questionnaire. This group was formed by enrolling volunteers from hospital staff. Racial backgrounds were all Japanese.

The diagnosis of AIP in all patients was based on criteria released by the Japan Pancreas Society using clinical data, imaging tests, and/or histopathological findings, as reported previously (25). High serum IgG4 concentrations (median, $730.0 \mathrm{mg} / \mathrm{dL}$; interquartile range, $265.0-1037.5 \mathrm{mg} / \mathrm{dL}$ ) were found in 55 of the 59 patients with AIP, and 52 of our 59 patients were treated with $40 \mathrm{mg}$ prednisolone daily for 4 weeks, which was then reduced by $5 \mathrm{mg}$ per week over a period of several weeks. All 52 AIP patients responded favorably to corticosteroid therapy, resulting in the improvement of clinical, laboratory, and imaging findings. Thirty-seven (63\%) had concurrent autoimmune diseases, including hypothyroidism (11 patients) and sclerosing cholangitis (34 patients), whose diagnosis was described in our prior studies $(11,26)$. We found no high concentrations of serum IgG4 in healthy subjects.

In total, we followed 55 patients, 52 of whom were treated with corticosteroids every month for a period of at least 12 months (median 72 months; 12-178 months). Patients underwent regular follow-up with an interview every month, laboratory tests every 2-3 months, and imaging tests, such as CT or MRI, every 6 months or in the event of relapse until October 2006. Sixteen of the $55(29 \%)$ patients relapsed during follow-up, which was defined as a recurrent attack of pancreatic swelling that resulted in irregular narrowing of the pancreatic duct or stenosis of the common bile duct, as reported previously (27).

All participants provided written informed consent for tests with DNA samples, and serum samples were obtained after receiving permission from patients. Serum samples were also collected from normal subjects after informed consent was obtained. This study has been approved by the institutional ethics committee.

\section{CTLA4 Genotyping}

Genomic DNA from patients and healthy individuals were isolated by phenolic extraction of sodium dodecyl sulfate-lysed and proteinase K-treated cells, as described previously $(28,29)$.

The five CTLA4 gene SNPs used in this study $(-1722,-658,-318,+49$, and +6230$)$ were genotyped using the 5' nucleae (TaqMan) assay using primers, probes, and reaction conditions as supplied by the manufacture (Applied Biosystems, Tokyo, Japan). The SNPs at $-1722,-658$, and -318 were located in the promoter region, the SNP at +49 was in exon 1 , and the SNP at +6230 was in the 3 ' untranslated region. Polymerase chain reaction was performed with a TaqMan Assay for Real-Time PCR (7500 Real Time PCR System; Applied Biosystems) following manufacturer's instructions.

\section{Haplotype-Genotype Estimation}

The R package 'haplo.stats' from the R Project for Statistical Computing (http://www.r-project.org/) was used to evaluate the haplotype structure of the five SNPs in CTLA4. Pair-wise linkage disequilibrium (LD) among the SNPs were measured in both 
patients and controls, as indicated by 'Lewontin's D' obtained in the 'genetics' R package from the R Project for Statistical Computing.

\section{HLA Typing}

HLA class I and II alleles were identified using a Micro SSP ${ }^{T M}$ DNA Typing Kit (One Lambda, Canoga Park, CA, USA) (30). DNA typing of DRB1 and DQB1 alleles was performed by polymerase chain reaction-restriction fragment length polymorphism analysis, as reported previously $(28,29)$.

\section{Laboratory Tests}

Serum levels of IgG4 were determined by single radial immunodiffusion kits (normal: $<135 \mathrm{mg} / \mathrm{dL}$ ) and circulating immune complexes $(\mathrm{ClC})$ (immune complex mRF "Nissui"; Nissui Pharmaceutical Co., Ltd., Tokyo, Japan) were measured as reported previously (13). Serum sCTLA4 levels were examined by commercially available ELISA kits (MedSystems Diagnostics, Vienna, Austria) following manufacturer's instructions.

\section{Statistical Analysis}

The significance of allele distribution between AIP patients and healthy subjects were tested using the $\chi^{2}$ test for two-by two or two-by-three comparisons. When the number of subjects was less than 5, Fisher's exact test was used. A threshold $P$ value of less than 0.05 , prior to correction for multiple hypotheses testing (Bonferroni correction), was considered as suggestive evidence of association. The Mann-Whitney $U$ test was used to analyze continuous variables. Spearman's rank order correlations were used to evaluate clinical features. Statistical analyses were performed using SigmaStat software (version 2.03; SPSS, Chicago, IL).

\section{RESULTS}

\section{CTLA4 Genotyping in Patients with AIP and Healthy Subjects}

A total of five SNPs in the CTLA4 gene were genotyped in patients and controls, and observed genotype frequencies were all in Hardy-Weinberg equilibrium. Analysis of allelic frequencies revealed a significant difference between AIP patients and healthy subjects for the SNP $+6230\left(\chi^{2}=7.50, P=0.024\right)$. Positivity for SNP $+6230 \mathrm{~A}$ was significantly decreased in patients with AIP (odds ratio [OR] $=0.40 ; 95 \%$ confidence interval $[\mathrm{Cl}], 0.21-0.78 ; P=0.011$ ) than in healthy subjects (Table 1 ), indicating a significant association of the $+6230 \mathrm{G} / \mathrm{G}$ genotype with AIP, which was significantly increased $(\mathrm{OR}=2.48 ; 95 \% \mathrm{Cl}, 1.28-4.81 ; P=0.011)$ in comparison with healthy controls (Table 1). No other SNPs were found to be significantly associated with AIP.

The pairwise LD mapping confirmed that these alleles were strong LD with each other in a narrow range, showing LD index (>0.8). Strong LD was indicated in the same block for AIP patients and controls. Seven unique SNP haplotypes were found altogether, and four of them had frequencies of $>5 \%$ (Table 2). Association analysis using haplotypes calculated by EM algorithms showed haplotype 2, which was the only SNP $+6230 \mathrm{~A}$, was associated with AIP resistance $(\mathrm{OR}=0.49 ; 95 \% \mathrm{Cl}, 0.29-0.83 ; P=0.011)$. None of the other haplotypes were associated with either susceptibility or resistance to AIP.

Since we previously reported that the HLA DRB $1{ }^{*} 0405-\mathrm{DQB} 1{ }^{*} 0401$ haplotype is associated with AIP (15), we further investigated the genetic association between this and 
CTLA4 SNPs in AIP patients. Analysis of allelic frequencies revealed no significant difference between patients with and without the HLA DRB $1{ }^{*} 0405-D Q B 1{ }^{*} 0401$ haplotype for +6230 SNPs $\left(\chi^{2}=0.54, P=0.76\right)$.

\section{Association between CTLA4 SNPs and Clinical Findings}

In this study, AIP was found to be associated with the autoimmune diseases of sclerosing cholangitis $(34 / 44 ; 77 \%)$ and hypothyroidism (11/50;22\%). Since autoimmune thyroid disease was previously associated with CTLA4 SNPs $(23,31)$, we evaluated whether the concurrent autoimmune diseases here were also associated with CTLA4 SNPs. However, we found no significant association between CTLA4 SNPs and the two diseases (data not shown). We further analyzed the relationship between CTLA4 SNPs and risk of AIP relapse. Sixteen out of $55(29 \%)$ patients experienced relapse during the follow-up period, and the $+49 \mathrm{~A} / \mathrm{A}$ and $+6230 \mathrm{~A} / \mathrm{A}$ genotypes were associated with an enhanced risk of relapse $(\mathrm{OR}=5.45 ; 95 \% \mathrm{Cl}, 1.12-26.55 ; P=0.038$ and $\mathrm{OR}=12.66$; $95 \% \mathrm{Cl}, 1.29-124.51 ; P=0.022$, respectively) (Table 3 ). In addition, analysis of allelic frequencies revealed a significant difference between patients with or without relapse for the SNP +6230 genotype $\left(\chi^{2}=7.23, P=0.027\right)$. However, there was no significant statistical haplotype association with the relapse of AIP.

\section{Detection of Serum SCTLA4 Levels in Patients with AIP}

Serum SCTLA4 levels were examined in 52 patients with AIP, and 32 healthy subjects. Median sCTLA4 levels were significantly higher in AIP patients $(8.9 \mathrm{ng} / \mathrm{mL}$; interquartile range [IQR], 5.8-10.7) than healthy subjects $(2.9 \mathrm{ng} / \mathrm{mL} ; \mathrm{IQR}, 2.1-3.8 ; P<$ $0.001)$ (Figure 1). Values higher than control mean plus 2 standard deviations $(5.7 \mathrm{ng} / \mathrm{mL})$ were considered to be elevated in this study. Patients with AIP $(77 \%)$ had a significantly higher incidence of elevated sCTLA4 levels than healthy controls $(0 \% ; P<0.001)$. Median serum sCTLA4 levels were similar in AIP patients with +6230A/A $(7.9 \mathrm{ng} / \mathrm{mL} ;$ IQR, 4.0-10.3), +6230A/G (8.9 ng/mL; IQR, 5.9-10.4), and +6230G/G (9.1 ng/mL; IQR, 5.8-11.1) $(P=0.87$; Figure 2).

Serum IgG4 concentrations and immune complex levels are closely associated with AIP, both clinical parameters were examined for their correlation with sCTLA4 levels in AIP patients. However, there were no significant correlations between SCTLA4 levels and IgG4 concentrations (correlation coefficient $-0.18, P=0.21$ ) or $\mathrm{CIC}$ levels (correlation coefficient $-0.028, P=0.85)$.

\section{DISCUSSION}

Thus far, several risk factors have been implicated in the etiology of AIP, including the HLA DRB $1{ }^{*} 0405-D Q B 1{ }^{*} 0401$ haplotype and polymorphisms of FCRL3 gene $(15,16)$. However, a number of other genes outside the major histocompatibility complex region may also play a role in susceptibility to AIP. One such candidate gene is CTLA4, which has been associated with increased risk for various autoimmune diseases (18).

Although most reported disease associations with CTLA4 SNPs have shown significantly higher frequencies of the $+49 \mathrm{G}$ allele in patients than in controls, our results showed that the +49 SNP was not associated with AIP. However, we were able to detect an association with the $+6230 \mathrm{G} / \mathrm{G}$ genotype in patients with AIP. Recent studies have shown the $G$ susceptibility allele to also be associated with Graves' disease, autoimmune 
hypothyroidism, and type 1 diabetes (23). Thus, our results suggest that the +6230 SNP may be functionally linked with susceptibility to AIP, though we can not exclude the possibility that the +6230 SNP may only be a linkage marker for an unidentified susceptibility gene for AIP on chromosome 2q33.

We also found that the CTLA4 haplotype may be an important determinant of AIP protection as well, since haplotype containing the $+6230 \mathrm{~A}$ allele was significantly associated with a 0.4 times less likelihood to develop AIP. Taken together, these results suggest that CTLA4+6230 SNP plays a pivotal role for both susceptibility to and protection from AIP in Japanese. Although the functional effects of CTLA4 SNPs are still uncertain, further investigation is needed to clarify this association from different ethnicities in Europe and the USA.

A prior study from our laboratory showed that 11 of $42(26 \%)$ patients relapsed during follow-up (27), though we could not make the characteristic findings or find reliable parameters in these patients at that time. After increasing the number of patients, we measured that 16 of $55(29 \%)$ patients relapsed during follow-up. We also found that patients who experienced relapse had high association with $+49 \mathrm{~A} / \mathrm{A}$ or $+6230 \mathrm{~A} / \mathrm{A}$ genotypes. However, as there was still a small number of patients in our cohort, it will be necessary to conclude this association following more studies with larger groups. No haplotype association was found in patients with relapse. Patients with AIP presented with sclerosing cholangitis and hypothyroidism, as reported previously $(11,26)$. Although some studies have shown that autoimmune thyroid disease is associated with CTLA4 SNPs $(23,31)$, we did not find any associations in our cohort. Hypothyroidism complicated with AIP may have different genetic background from autoimmune thyroid disease.

Previously, Oaks et al. reported that SCTLA4 immunoreactivity was inhibited by binding to CD80 ligand, implying that SCTLA4 was indeed acting as a functional receptor (20). They reported that serum sCTLA4 levels were higher in patients with autoimmune thyroid disease than in healthy volunteers, in which sCTLA4 was not detectable. However, they also suggested that the SCLTA4 molecule may have a dual role by blocking the interaction of CD80 on antigen-presenting cells and mCTLA4 on T cells and interfering with the inhibitory signal sending to T cells, thus enhancing immune responses. In another report, Magistrelli et al. (32) reported that sCTLA4 was detectable in 14 of 64 healthy subjects. Our study clearly demonstrated that patients with AIP have significantly increased sCTLA4 levels in serum and higher overall incidences of elevated sCTLA4. Due to the fact that we found no correlation between sCTLA4 and IgG4 level, which is associated with disease activity, we could not conclude whether increased SCTLA4 levels are beneficial or harmful to patients with AIP. Further longitudinal follow-up studies are required to resolve this critical question.

Recently, functional analyses revealed the existence of two mRNA CTLA4 isoforms: a full length isoform (flCTLA4) encoded by exons 1-4, and a sCTLA4 that lacks exon 3 (23). The ratio of sCTLA4 to fICTLA4 mRNA splice forms in unstimulated CD4 T cells was $50 \%$ lower in $+6230 \mathrm{G} / \mathrm{G}$ positive disease-susceptible individuals compared with A/A protected individuals, suggesting that this $6.1 \mathrm{~kb}$ region determines splicing efficiency and production of SCTLA4, with the $+6230 \mathrm{G}$ disease-susceptibility haplotype producing less sCTLA4 transcripts than the $+6230 \mathrm{~A}$ haplotype. However, Anjos et al. (33) reported no observable effect of the +6230 SNP on the expression of either SCTLA4 or fICTLA4 
using allele-specific single-nucleotide primer extensions. We also failed to find a correlation between +6230 alleles and serum sCTLA4 levels, regardless of susceptibility to AIP in $+6230 \mathrm{G} / \mathrm{G}$. These conflicting interpretations may be accounted by patient and control selections, sample size differences, and/or technical variances, and a reasonable explanation for the increased SCTLA4 in patients with AIP needs further exploration.

As previous studies have reported that serum IgG4 concentrations and $\mathrm{CIC}$ levels are closely associated with AIP (13), we therefore examined the correlation between these indicators and CTLA4 SNPs in our AIP patients, but could find no correlation. We had reported in an earlier study that $F C R L 3+110$ SNP was positively correlated with serum IgG4 concentrations and CIC levels in patients with AIP (16). Since FCRL3 shows high structural homology with classical Fcy receptor genes, the +110 SNP might have accounted for the associated with IgG4 secretion in AIP. CTLA4 is expressed on T cell surfaces and plays a role as a negative regulator of $T$ cell responses, so the lack of association between CTLA4+6230 SNP and serum IgG4 concentrations and CIC levels is understandable.

Lastly, although the HLA DRB1*0405-DQB1*0401 haplotype and CTLA4+6230 SNP both indicated susceptibility to AIP, we showed that there was no genetic association between these two markers in this study. These data suggest that the mechanisms by which HLA and CTLA4 induce AIP are different. Since HLA-DR genes and CTLA4 gene are located on different chromosomes, there is no genetical linkage between these genes.

In conclusion, AIP is closely associated with the CTLA4+6230 SNP and serum sCTLA4 levels. The CTLA4 gene might play a crucial role in the pathogenesis of this multi-factorial disease, and further studies are needed to clarify its role in AIP.

\section{ACKNOWLEDGEMENTS:}

The authors would like to thank Yuki Akahane, Asami Yamazaki, and Toyo Amaki for their technical assistance, and Trevor Ralph for his editorial assistance.

Guarantor of the article: Takeji Umemura, M.D., Ph.D.

Specific author contributions: Takeji Umemura: study design, assays, data analysis, statistical analysis, drafting of manuscript. Masao Ota: study design, data analysis, drafting manuscript. Hideaki Hamano: material collection, data analysis, manuscript review. Yoshihiko Katsuyama: assays, data analysis, manuscript review. Takashi Muraki: material collection, data analysis, manuscript review. Norikazu Arakura: material collection, manuscript review. Shigeyuki Kawa: study design, material collection, data analysis, supervision, drafting of manuscript. Kendo Kiyosawa: data analysis, supervision, manuscript review.

Financial support: Professor Shigeyuki Kawa - Supported in part by Grants-in-Aid for Scientific Research from the Ministry of Education, Science, Sports and Culture of Japan (12670471, 13557047, 15659167 and 16390205) and Japan Health Sciences Foundation (KH21022), and by a Research of Specific Diseases, Health and Labour Sciences Research Grant, Japan. Dr. Takeji Umemura - Supported by the Foundation of Nagano Prefecture for Promoting Science.

Potential competing interests: None 


\section{REFERENCES}

1. Yoshida K, Toki F, Takeuchi T, et al. Chronic pancreatitis caused by an autoimmune abnormality. Proposal of the concept of autoimmune pancreatitis. Dig Dis Sci 1995;40:1561-8.

2. Horiuchi A, Kaneko T, Yamamura N, et al. Autoimmune chronic pancreatitis simulating pancreatic lymphoma. Am J Gastroenterol 1996;91:2607-9.

3. Ito T, Nakano I, Koyanagi S, et al. Autoimmune pancreatitis as a new clinical entity. Three cases of autoimmune pancreatitis with effective steroid therapy. Dig Dis Sci 1997;42:1458-68.

4. Horiuchi A, Kawa S, Akamatsu T, et al. Characteristic pancreatic duct appearance in autoimmune chronic pancreatitis: a case report and review of the Japanese literature. Am J Gastroenterol 1998;93:260-3.

5. $\quad$ Okazaki K, Chiba T. Autoimmune related pancreatitis. Gut 2002;51:1-4.

6. Horiuchi A, Kawa S, Hamano H, et al. ERCP features in 27 patients with autoimmune pancreatitis. Gastrointest Endosc 2002;55:494-9.

7. Hamano $\mathrm{H}$, Kawa $\mathrm{S}$, Ochi $\mathrm{Y}$, et al. Hydronephrosis associated with retroperitoneal fibrosis and sclerosing pancreatitis. Lancet 2002;359:1403-4.

8. Zen Y, Harada K, Sasaki M, et al. IgG4-related sclerosing cholangitis with and without hepatic inflammatory pseudotumor, and sclerosing pancreatitis-associated sclerosing cholangitis: do they belong to a spectrum of sclerosing pancreatitis? Am J Surg Pathol 2004;28:1193-203.

9. Chari ST, Smyrk TC, Levy MJ, et al. Diagnosis of autoimmune pancreatitis: the mayo clinic experience. Clin Gastroenterol Hepatol 2006;4:1010-6.

10. Deshpande V, Chicano S, Finkelberg D, et al. Autoimmune pancreatitis: a systemic immune complex mediated disease. Am J Surg Pathol 2006;30:1537-45.

11. Hamano $\mathrm{H}$, Arakura N, Muraki T, et al. Prevalence and distribution of extrapancreatic lesions complicating autoimmune pancreatitis. J Gastroenterol 2006;41:1197-205.

12. Umemura T, Zen $\mathrm{Y}$, Hamano H, et al. IgG4-Hepatopathy: Association of IgG4-bearing plasma cells in liver with autoimmune pancreatitis. Hepatology;(in press).

13. Hamano $\mathrm{H}$, Kawa S, Horiuchi A, et al. High serum IgG4 concentrations in patients with sclerosing pancreatitis. N Engl J Med 2001;344:732-8.

14. Aparisi L, Farre A, Gomez-Cambronero L, et al. Antibodies to carbonic anhydrase and IgG4 levels in idiopathic chronic pancreatitis: relevance for diagnosis of autoimmune pancreatitis. Gut 2005;54:703-9.

15. Kawa S, Ota M, Yoshizawa K, et al. HLA DRB10405-DQB10401 haplotype is associated with autoimmune pancreatitis in the Japanese population. Gastroenterology 2002;122:1264-9.

16. Umemura $\mathrm{T}$, Ota $\mathrm{M}$, Hamano $\mathrm{H}$, et al. Genetic association of Fc receptor-like 3 polymorphisms with autoimmune pancreatitis in Japanese patients. Gut 2006;55:1367-8.

17. Kamisawa T, Funata N, Hayashi Y, et al. Close relationship between autoimmune pancreatitis and multifocal fibrosclerosis. Gut 2003;52:683-7.

18. Gough SC, Walker LS, Sansom DM. CTLA4 gene polymorphism and autoimmunity. Immunol Rev 2005;204:102-15.

19. Tivol EA, Borriello F, Schweitzer AN, et al. Loss of CTLA-4 leads to massive lymphoproliferation and fatal multiorgan tissue destruction, revealing a critical negative regulatory role of CTLA-4. Immunity 1995;3:541-7.

20. Oaks MK, Hallett KM. Cutting edge: a soluble form of CTLA-4 in patients with autoimmune thyroid disease. J Immunol 2000;164:5015-8.

21. Liu MF, Wang CR, Chen PC, et al. Increased expression of soluble cytotoxic T-lymphocyte-associated antigen-4 molecule in patients with systemic lupus erythematosus. Scand J Immunol 2003;57:568-72.

22. Wang XB, Kakoulidou M, Giscombe R, et al. Abnormal expression of CTLA-4 by T cells from patients with myasthenia gravis: effect of an AT-rich gene sequence. J Neuroimmunol 2002;130:224-32. 
23. Ueda $\mathrm{H}$, Howson JM, Esposito $\mathrm{L}$, et al. Association of the T-cell regulatory gene CTLA4 with susceptibility to autoimmune disease. Nature 2003;423:506-11.

24. Thio CL, Mosbruger TL, Kaslow RA, et al. Cytotoxic T-lymphocyte antigen 4 gene and recovery from hepatitis B virus infection. J Virol 2004;78:11258-62.

25. Pearson RK, Longnecker DS, Chari ST, et al. Controversies in clinical pancreatology: autoimmune pancreatitis: does it exist? Pancreas 2003;27:1-13.

26. Komatsu K, Hamano H, Ochi $\mathrm{Y}$, et al. High prevalence of hypothyroidism in patients with autoimmune pancreatitis. Dig Dis Sci 2005;50:1052-7.

27. Takayama M, Hamano $\mathrm{H}$, Ochi $\mathrm{Y}$, et al. Recurrent attacks of autoimmune pancreatitis result in pancreatic stone formation. Am J Gastroenterol 2004;99:932-7.

28. Ota M, Seki T, Nomura N, et al. Modified PCR-RFLP method for HLA-DPB1 and -DQA1 genotyping. Tissue Antigens 1991;38:60-71.

29. Ota M, Seki T, Fukushima H, et al. HLA-DRB1 genotyping by modified PCR-RFLP method combined with group-specific primers. Tissue Antigens 1992;39:187-202.

30. Umemura T, Ota M, Yoshizawa K, et al. Lack of association between FCRL3 and FcgammaRII polymorphisms in Japanese type 1 autoimmune hepatitis. Clin Immunol 2007; 122:338-42.

31. Furugaki K, Shirasawa S, Ishikawa N, et al. Association of the T-cell regulatory gene CTLA4 with Graves' disease and autoimmune thyroid disease in the Japanese. J Hum Genet 2004;49:166-8.

32. Magistrelli $G$, Jeannin P, Herbault N, et al. A soluble form of CTLA-4 generated by alternative splicing is expressed by nonstimulated human $\mathrm{T}$ cells. Eur $\mathrm{J}$ Immunol 1999;29:3596-602.

33. Anjos SM, Shao W, Marchand $L$, et al. Allelic effects on gene regulation at the autoimmunity-predisposing CTLA4 locus: a re-evaluation of the $3^{\prime}+6230 \mathrm{G}>\mathrm{A}$ polymorphism. Genes Immun 2005;6:305-11. 
1 Table 1. CTLA4 Polymorphisms in 59 Patients with AIP and 102 Healthy Subjects

\begin{tabular}{|c|c|c|c|c|}
\hline & & dency (\%) & & \\
\hline & $\begin{array}{c}\text { AIP } \\
(n=59)\end{array}$ & $\begin{array}{l}\text { Controls } \\
(n=102)\end{array}$ & $P$ value (OR: $95 \% \mathrm{Cl}$ ) & $P c$ \\
\hline+6230 & & & & \\
\hline Genotype frequen & & & & \\
\hline $\mathrm{A} / \mathrm{A}$ & 8.5 & 11.8 & 0.70 & \\
\hline$A / G$ & 27.1 & 46.1 & 0.027 (0.44: $0.22-0.87)$ & 0.14 \\
\hline G/G & 64.4 & 42.2 & 0.011 (2.48: 1.28-4.81) & 0.055 \\
\hline Allele carrier frequ & & & & \\
\hline$A(A / A+A / G)$ & 35.6 & 57.8 & $0.011(0.40: 0.21-0.78)$ & \\
\hline$G(A / G+G / G)$ & 91.5 & 88.2 & 0.70 & \\
\hline Allele frequency & & & & \\
\hline A allele & 22.0 & 34.8 & 0.023 (0.53: 0.31-0.89) & \\
\hline G allele & 78.0 & 65.2 & 0.023 (1.89: 1.12-3.18) & \\
\hline-1722 & & & & \\
\hline C allele & 39.0 & 35.3 & 0.15 & \\
\hline T allele & 61.0 & 64.7 & - & \\
\hline-658 & & & & \\
\hline T allele & 0.0 & 0.0 & 1.00 & \\
\hline C allele & 100.0 & 100.0 & - & \\
\hline-318 & & & & \\
\hline
\end{tabular}




$\begin{array}{cccc}\text { T allele } & 14.4 & 9.8 & 1.00 \\ \text { C allele } & 85.6 & 90.2 & - \\ +49 & & & \\ \text { A allele } & 34.7 & 44.6 & 0.33 \\ \text { G allele } & 65.3 & 55.4 & -\end{array}$

1 Abbreviations: AIP, autoimmune pancreatitis; OR, odds ratio; Cl, confidence interval.

$2 \quad$ PC value was detected after a Bonferroni correction. 
Table 2. CTLA4 Haplotypes in Patients with AIP and Healthy Subjects

\begin{tabular}{|c|c|c|c|c|c|c|c|c|c|}
\hline \multirow{3}{*}{ Haplotype } & \multicolumn{5}{|c|}{ SNP at position: } & \multicolumn{2}{|c|}{ Proportion of indicated haplotype (\%) } & \multirow{3}{*}{$P$ value (OR:95\% Cl) } & \multirow{3}{*}{$\begin{array}{l}\text { Hapmap } \\
(n=54)\end{array}$} \\
\hline & & & & & & AIP & Controls & & \\
\hline & -1722 & -658 & -318 & +49 & +6230 & $(n=118)$ & $(n=204)$ & & \\
\hline 1 & $\mathrm{C}$ & $\mathrm{C}$ & $\mathrm{C}$ & $\mathrm{G}$ & $\mathrm{G}$ & 39.0 & 34.7 & 0.53 & 38.6 \\
\hline 2 & $\mathrm{~T}$ & C & C & A & A & 20.3 & 34.2 & 0.011 (0.49: 0.29-0.83) & 29.5 \\
\hline 3 & $\mathrm{~T}$ & C & C & G & G & 26.3 & 20.7 & 0.30 & 25.0 \\
\hline 4 & $\mathrm{~T}$ & $\mathrm{C}$ & $\mathrm{T}$ & A & G & 12.7 & 9.8 & 0.53 & 6.8 \\
\hline
\end{tabular}

2 Abbreviations: AIP, autoimmune pancreatitis; OR, odds ratio; $\mathrm{Cl}$, confidence interval.

3 Values for $\mathrm{n}$ indicate two times the number of individuals since each person carries two haplotypes.

4 Hapmap data indicate the 4 haplotype-tagging SNPs at $-1722,-658,-318$, and +49 . 
1 Table 3. CTLA4 +49 and +6230 Polymorphisms and Relapse of AIP

\begin{tabular}{|c|c|c|c|}
\hline & \multicolumn{2}{|c|}{ Frequency (\%) } & \multirow[b]{2}{*}{$P$ value (OR: $95 \% \mathrm{Cl}$ ) } \\
\hline & $\begin{array}{c}\text { Relapse }(+) \\
\qquad(\mathrm{n}=16)\end{array}$ & $\begin{array}{l}\text { Relapse (-) } \\
\qquad(\mathrm{n}=39)\end{array}$ & \\
\hline$+49 \mathrm{~A} / \mathrm{A}$ & 31.3 & 7.7 & $0.038(5.45: 1.12-26.55)^{\star}$ \\
\hline$+49 A / G$ & 25.0 & 48.7 & 0.14 \\
\hline$+49 G / G$ & 43.8 & 43.6 & 0.77 \\
\hline$+6230 \mathrm{~A} / \mathrm{A}$ & 25.0 & 2.6 & 0.022 (12.66: 1.29-124.51)* \\
\hline$+6230 \mathrm{~A} / \mathrm{G}$ & 18.8 & 33.3 & 0.34 \\
\hline$+6230 \mathrm{G} / \mathrm{G}$ & 56.3 & 64.1 & 0.81 \\
\hline
\end{tabular}

2 Abbreviations: AIP, autoimmune pancreatitis; OR, odds ratio; $\mathrm{Cl}$, confidence interval.

$3 \quad$ * Fisher's exact test. 


\section{Figure Legends}

Figure 1. Detection of serum SCTLA4 levels in patients with AIP, and healthy controls.

Serum SCTLA4 levels were detected in 52 patients with AIP, and 32 healthy subjects. Solid lines indicate median values. The broken line indicates the cutoff value (control group mean +2 standard deviations).

AIP, autoimmune pancreatitis.

Figure 2. CTLA4+6230 SNPs and serum SCTLA4 levels in patients with AIP. Serum sCTLA4 levels were detected in 4 patients with the +6230A/A SNP, 16 patients with the $+6230 \mathrm{~A} / \mathrm{G}$ SNP, and 32 patients with the $+6230 \mathrm{G} / \mathrm{G}$ SNP. Solid lines indicate median values.

AIP, autoimmune pancreatitis. 
Figure 1.

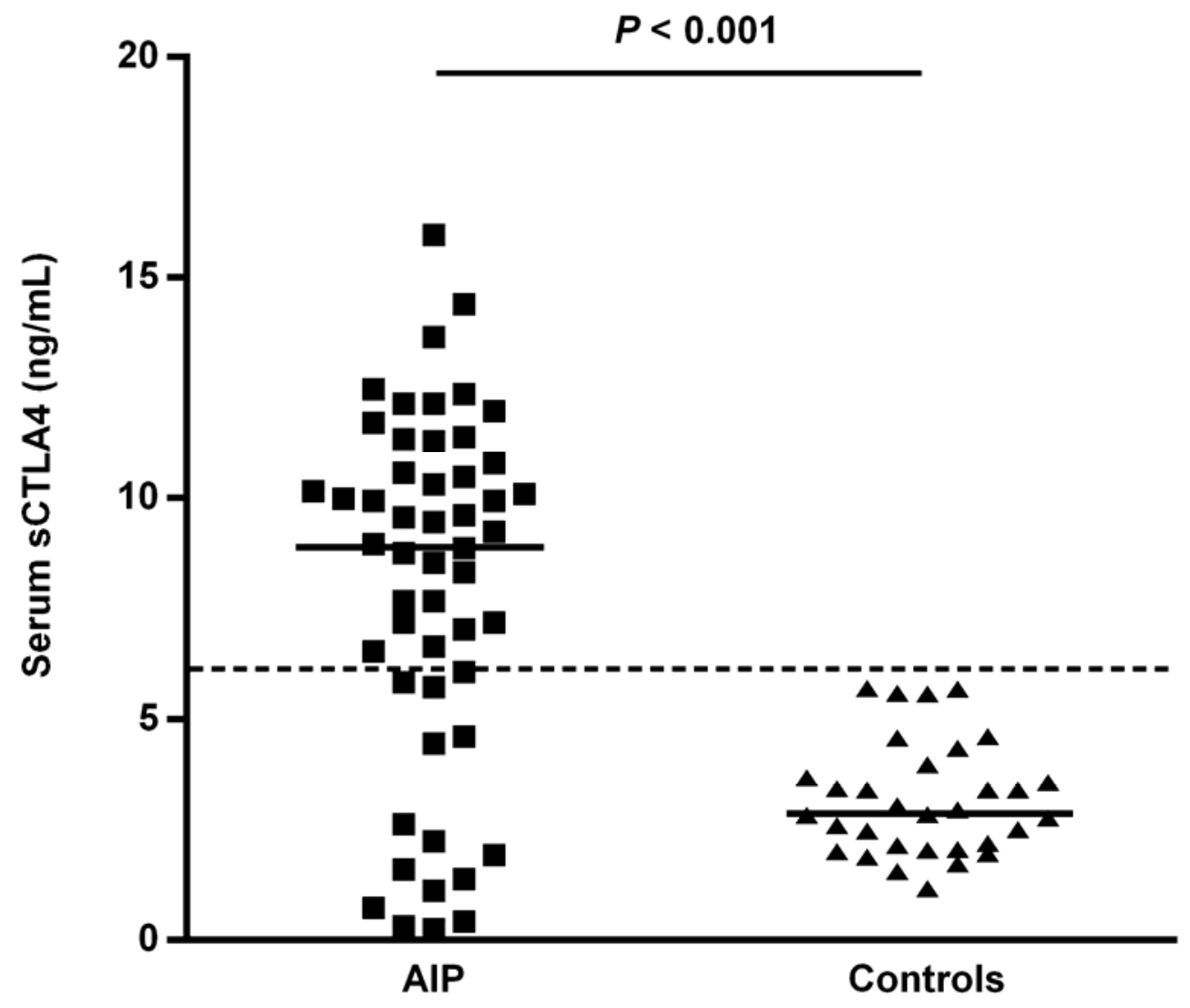


Figure 2.

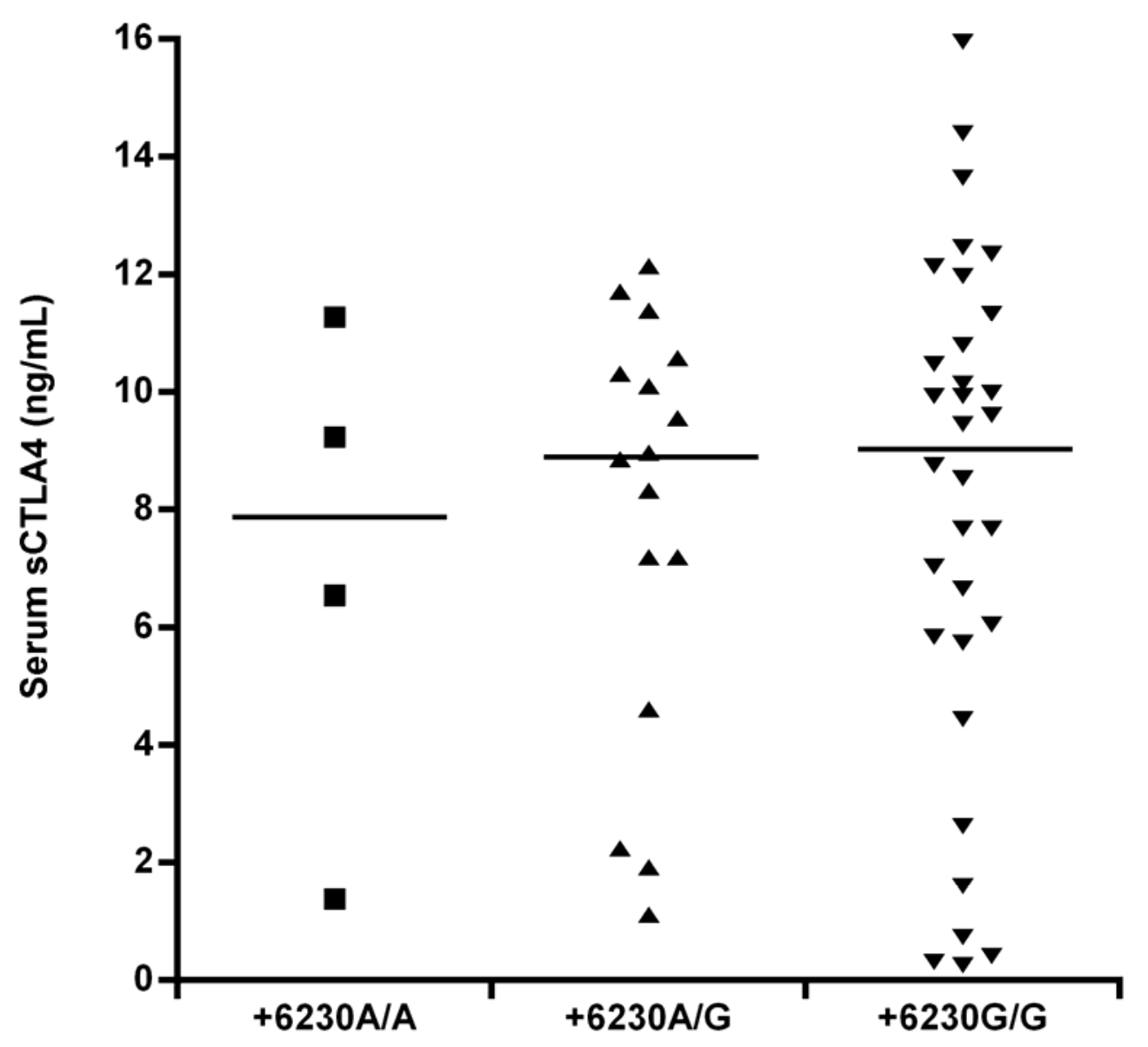

